\title{
Studies on structural elucidation of delphinium alkaloids by using LC- ESI-MS technique.
}

\author{
Bilal Ahmad Dar, Mushtaq Ahmad Qurishi, Aijaz Hussain Kanth \\ University of Kashmir, Hazratbal, Srinagar, Kashmir, India. \\ darbilal29@yahoo.in \\ University of Kashmir, Hazratbal, Srinagar, Kashmir, India. \\ qurishi@gmail.com \\ Department of chemistry GDC Bemina, Srinagar, Kashmir.
}

Aijazkanth@rediffmail.com

\begin{abstract}
1. A rapid,sensitive and specific liquid chromatography-electron spray-mass spectrometry (LC-ESI-MS) method to identify the different diester-diterpenoid and monoester type alkaloids from delphinium cashmerianum Collected from Sopi Kargil. Chromatographic separation were achieved on c-18 coloumn and peaks detemined by mass spectrometry in positive and negative modes.The validated method led to tentative identification of eight alkaloids on the basis of their retention times and fragmentation patterns. Results showed that the positive mode response was much higher than the negative ion mode .chromatographic conditions were optimized to obtain high resolution and short run time.
\end{abstract}

\section{Indexing terms/Keywords}

delphinium cashmerianum, LC-ESI-MS, Sopi.

\section{Academic Discipline and Sub-Disciplines}

Applied material Sciences.

\section{SUBJECT CLASSIFICATION}

Phytochmistry subject classification.

\section{TYPE (METHOD/APPROACH)}

Experimental. Analytical liquid chromatographic and mass spectrometric method.

\section{Council for Innovative Research}

Peer Review Research Publishing System

Journal: Journal of Advances in Chemistry

Vol. 11, No. 3

editorjaconline@gmail.com

www.cirjac.com 


\section{INTRODUCTION}

It is the genus belonging to family renunculaceae comprising of more than 300 species of perennial flowering plants distributed in Asia, North America, Europe and tropical Africa ${ }^{1}$.The members of this genus are toxic to humans and livestock $^{2}$. The perennial species of Delphinium along with the annual species of consolidate are commonly called as Larkspur $^{3,4}$. In the flowering seas on of June - July the plant has raceme of colored flowers varying from purple to blue and red and white. In most species the flower has five petal like sepals joined together with a spur(hence common name) enclosing four similarly colored petals. They possess small black and shiny seeds.

Delphinium cashmerianum Royle (Kashmir Larkspur) is a perennial herb found in the Himalayas, from Pakistan to Uttarakhand, at altitudes of $2700-4500 \mathrm{~m}$. It is characterized by woolly- haired, conspicuously veined bluish-purple flowers, $2-3 \mathrm{~cm}$ across clustered in a dense flat-topped head. Flowers have a stout spur $1.5 \mathrm{~cm}$ long. Leaves are rounded in outline, deeply lobed, $3-5 \mathrm{~cm}$ across. It is quite similar to Musk Larkspur which is found only above altitudes of $4500 \mathrm{~m}$, and has larger, more inflated flowers.

The majority of phytochemical investigation on Delphinium species (Renunculaceae) have been carried out for diterpenoidalkaloid.AlkaloidslikeNeoline,Chasmanine,Homochasmanineand Delphisine were reported by pelletier ${ }^{5}$ in Delphinium staphisagria in 1975 . Warnock ${ }^{6}$ reported benzoylated quercetin glycosides from Delphinium carolinianum in 1982. Jong-C Park and S. W Pelletier ${ }^{7}$ is olated hetisane type of alkaloids like davisinol, 18 benzoyldavisinol and davisin from Delphinium davisii. Gabriel De La Fuente ${ }^{8}$ reported is olation of six new norditerpenoid alkaloids Nudicaulidines from Delphinium cardiopetalum. Ayhan Ulubelen ${ }^{9}$ et al have reported norditerpenoid 8 -acetyl condelphine, condelphine and senbusine from Delphinium pyrimadale.Delavaine, deoxylycoctinine and methyllycaconitine from Delphinium disectum were reported by Nyamdari Batbayar et $\mathrm{al}^{10}$. Werner Herz et $\mathrm{al}^{11}$ have reported flavonol glycosides from ethanolic extract of aerial parts of Delphinium staphisagria.Yang-Qing $\mathrm{He}$ et $\mathrm{al}^{12}$ have also reported flavanoids from Delphinium albocoeruleum along with diterpenoids and one triterpenoid.

\section{MATERIALS AND METHODS Plant Material}

The root part of Delphinium cashmerianum (15 Kg) were collected from SOPI, Kargil Ladakh (J\&K, India) in august 2010. The specimen was identified by Akhtar H. Malik, Curator, Centre for Biodiversity \& Taxanomy, University of Kashmir (Specimen deposited under accession No. 341320 and Collection No. 1301 - Bilal, Kash).

\section{Extraction}

The air dried, finely powdered root material $(5 \mathrm{Kg})$ was extracted for 72 hours with methanol to afford the respective extract, which was concentrated under reduced pressure and was coded as DEL.

\section{Reagents and chemicals}

HPLC grade acetonitrile, methanol and formic acid were purchased from Sigma Aldrich. HPLC grade water (18.2M 2 ) was procured from Milli- Q water system(USA). other chemicals were of analytical grade and are available commercially.

\section{LC System}

LC analysis was carried out by an Agilent1260 infinity series consisting of a pump, detector, an auto sampler and a column component. The samples were separated on chromo lith RP-18e column (4.6 mm ID, 50mm length) Merck at room temperature. The mobile phase consisted of $(A)$ aqueous formic acid $(0.1 \%)$ and $(B)$ methanol and the elution gradient was set as follows : ; 0-8 min, linear gradient from $12 \%$ to $25 \%$ of $B ; 8-12 \mathrm{~min}$, is ocratic conditions at $25 \%$ of $B$;

$12-16 \mathrm{~min}$, linear gradient from $25 \%$ to $40 \%$ of $\mathrm{B} ; 16-40 \mathrm{~min}$, linear gradient from 40 to $50 \%$ of $\mathrm{B}, 40-50 \mathrm{~min}$, linear gradient from 50 to $100 \%$ of $B$. Flow rate: $1 \mathrm{ml} / \mathrm{min}$.

\section{LIQUID-CHROMATOGRAPHY TANDEM-MASS-SPECTROMETRY (LC-ESI-MSMS)}

For LC-ESI-MS ${ }^{n}$ experiments, LC-MS QqQ-6410B equipment (Agilent Technologies) comprising a chromatographic system 1260 Infinity (Agilent Technologies) coupled with an Agilent Triple Quad mass spectrometer fitted with an ESI source was used. The conditions of ESI source were set as follows: sheath and auxillary gases flow, 25 and 3 arbitrary units, respectively; spray voltage, $4 \mathrm{KV}$; capillary temperature $325^{\circ} \mathrm{C}$ and tube length voltage: $120 \mathrm{~V}$. The samples were analysed in both positive and negative modes and full scan mass range was set between $\mathrm{m} / \mathrm{z} 100-1200$ with the aquisition of centroided-type mass spectra. Accurate mass analyses were calibrated according to manufacturers guidelines. In the $M^{n}$ experiments, data dependent $\mathrm{MS}^{n}$ scanning was performed to minimize total analysis time as it can trigger fragmentation spectra of target ions and prevent repitition by dynamic exclusion settings. The software of the Agilent technolgies was used for data analysis. After the automated removal of noise and baseline signals, the theoritical extract chromatogram of main alkaloids was shown in the plot (fig 3) 


$\begin{array}{llll}\text { Data Filename } & \text { DELKASH5.2MG-1.4ML0004.d } & \text { Sample Name } & \text { DELKASH5.2MG-1.4ML } \\ \text { Sample Type } & \text { Sample } & \begin{array}{l}\text { Position } \\ \text { User Name }\end{array} & \text { Vial } 47 \\ \begin{array}{l}\text { Instrument Name } \\ \text { Acq Method }\end{array} & \text { SG11351102 } & \text { Acquired Time } & 5 / 4 / 20131: 32: 24 \text { PM } \\ \text { IRM Calibration Status } & \text { Not Applicable } & \text { DA Method } & \text { ARTEMISIN-MS2.m } \\ \text { Comment } & \text { 50MM+100MMX4.9MM CHROMO } & & \end{array}$

Sample Group Info.

User Defined $1 \quad$ User Defined 2

User Chromatograms

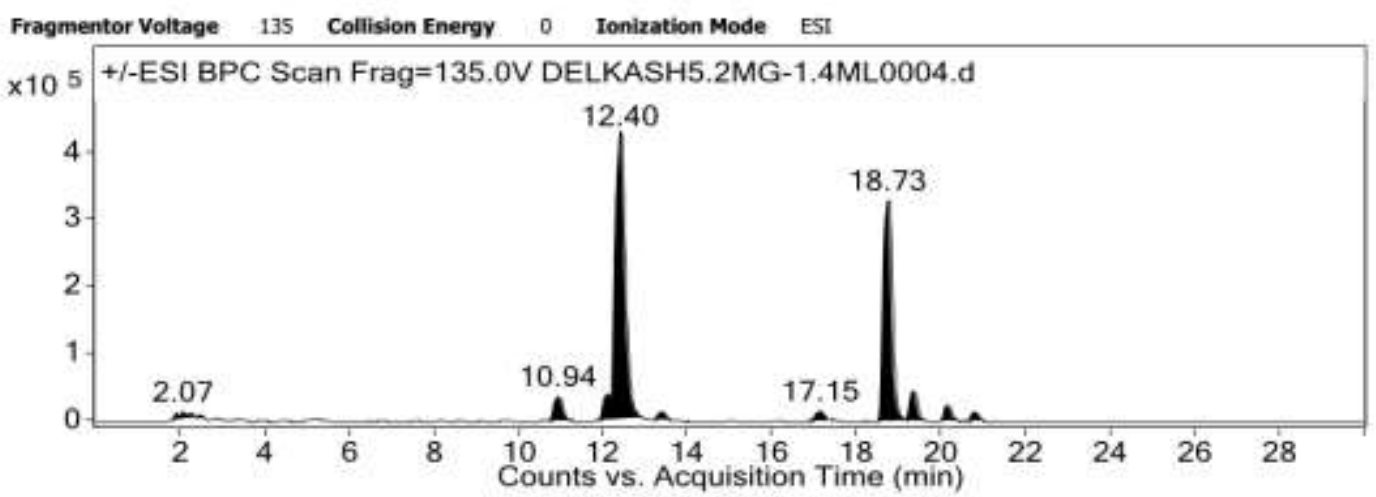

\begin{tabular}{|c|c|c|c|c|}
\hline Start & RT & End & Height & Area \\
\hline 10.73 & 10,94 & 11.32 & 35880 & 487068 \\
\hline 11.87 & 12.11 & 12.18 & 39047 & 410807 \\
\hline 12,18 & 12.4 & 12.93 & 429546 & 6816676 \\
\hline 13.21 & 13.36 & 13.37 & 10283 & 50384 \\
\hline 13,37 & 13.39 & 13.42 & 10318 & 30032 \\
\hline 17.06 & 17.15 & 17.31 & 14325 & 142471 \\
\hline 18.5 & 18.73 & 19.13 & 330205 & 4569394 \\
\hline 19.17 & 19.33 & 19.63 & 44140 & 510437 \\
\hline 19.99 & 20.15 & 20.41 & 22409 & 249431 \\
\hline 20.62 & 20.78 & 21.01 & 12586 & 139651 \\
\hline
\end{tabular}

fig-3 Total chromatogram of Delphinium cashmerianum.

\section{RESULTS AND DISCUSSION}

For LC-MS experiments different fragmentation measurements were conducted in independent LC-MS ${ }^{\mathrm{n}}$ experiments. In the first measurement the survey scan was performed in analyser at $(R=30,000)$ followed by $M^{2}{ }^{2}$ scan at $(R=15,000)$. The accurate tandem mass spectra can provide strong evidences for identification of fragments and neutral loss. In the separate measurement in which ESI $\mathrm{MS}^{2}$ and $\mathrm{MS}^{3}$ experimens using dynode detection provided more fragment ions and order of fragmentation could be determined to show evidence of structural identification. Fifteen alkaloids were picked from the chromatogram at the retention times from $2-20 \mathrm{~min}$. Among them nine compounds were identified as 14-2methylbutylnudicaudiline, 14-cis cinnamoyl nudicaudiline, Davisinol, uncinitine, condelphine, Peregrine, Karakoline and 10- hydroxy methyllyoaconitine. by comparing their retention times and on the bas is of their fragmentation patterns in MSMS data. The other compounds were tentatively identified by comparing their mass data with those reported in literature. The Retention time values and mass data of deduced compounds from peaks are summarised in table 3 . The fragmentation patterns of the identified compounds is shown in (fig 4). 
Table-3

\begin{tabular}{|c|c|c|c|c|c|}
\hline $\begin{array}{l}\mathrm{S} \\
\mathrm{N} \\
\mathrm{O}\end{array}$ & $\begin{array}{l}\text { Trin } \\
\text { minute } \\
\text { s }\end{array}$ & $\mathrm{m} / \mathrm{z}$ & Adduct peaks & $\begin{array}{l}\text { Fragmentation } \\
\text { peaks }\end{array}$ & Tdentification \\
\hline 1 & $\begin{array}{l}2.0- \\
2.10\end{array}$ & $507\left[\mathrm{C}_{28} \mathrm{H}_{45} \mathrm{NO}_{7}\right]$ & $[\mathrm{M}+2] 509,[\mathrm{M}+2 \mathrm{H}+\mathrm{Na}] 533]$ & $\mathrm{MS}^{<}[533] 428,365$ & 14-Isobutylnudicaudiline \\
\hline 2 & $\begin{array}{l}2.29- \\
2.36\end{array}$ & $521\left[\mathrm{C}_{29} \mathrm{H}_{47} \mathrm{NO}_{7}\right]$ & {$[\mathrm{M}+2] 523,[\mathrm{M}+3] 524$} & $\mathrm{MS}^{<}[523] 443,365$ & $\begin{array}{l}\text { 14-(2- } \\
\text { methylbutylnudicaudiline. }\end{array}$ \\
\hline 3 & $\begin{array}{l}10.80- \\
11.13\end{array}$ & $313\left[\mathrm{C}_{20} \mathrm{H}_{27} \mathrm{NO}_{7}\right]$ & $\begin{array}{l}{[\mathrm{M}+1] 314,[\mathrm{M}+2] 315,[\mathrm{M}+3] 316,} \\
{[\mathrm{M}-1] 312}\end{array}$ & $\mathrm{MS}^{2}[315] 271$ & Davisinol \\
\hline 4 & $\begin{array}{l}12.24- \\
12.63\end{array}$ & $359\left[\mathrm{C}_{22} \mathrm{H}_{33} \mathrm{NO}_{3}\right]$ & $[\mathrm{M}-1] 358],[\mathrm{M}+2 \mathrm{H}+\mathrm{Na}] 386$ & $\mathrm{MS}^{\mathrm{2}}[359] 341,297$ & Uncinitine \\
\hline 5 & $\begin{array}{l}13.26- \\
13.37\end{array}$ & $445\left[\mathrm{C}_{26} \mathrm{H}_{39} \mathrm{NO}_{5}\right]$ & $\begin{array}{l}[\mathrm{M}+\mathrm{H}+\mathrm{Na}] 469],[\mathrm{M}+\mathrm{CAN}+\mathrm{Na}] 5 \\
10\end{array}$ & $\begin{array}{l}\mathrm{MS}^{2}[448] 360,342, \\
308\end{array}$ & Peregrine \\
\hline 6 & $\begin{array}{l}13.42- \\
13.55\end{array}$ & $449\left[\mathrm{C}_{25} \mathrm{H}_{39} \mathrm{NO}_{6}\right]$ & {$[\mathrm{M}+19] 468$} & $\begin{array}{l}\mathrm{MS}^{<}[449] 359,341,31 \\
4\end{array}$ & Condelphine \\
\hline 7 & $\begin{array}{l}17.06- \\
17.53\end{array}$ & $374\left[\mathrm{C}_{22} \mathrm{H}_{32} \mathrm{NO}_{4}\right]$ & {$[\mathrm{M}+2] 376,[\mathrm{M}-18] 356$} & $\mathrm{MS}^{2}[374] 356,324$, & Karakoline \\
\hline 8 & $\begin{array}{l}19.21 \\
- \\
19.50\end{array}$ & $\begin{array}{l}698\left[\mathrm{C}_{37} \mathrm{H}_{50} \mathrm{~N}_{2} \mathrm{O}_{1}\right. \\
\text { 1] }\end{array}$ & $\begin{array}{l}{[\mathrm{M}+1] 699,[\mathrm{M}+2] 700,[\mathrm{M}+24] 72} \\
3\end{array}$ & $\mathrm{MS}^{2}[700] 683,587$ & $\begin{array}{l}10- \\
\text { Hydroxymethyllyoaconitin } \\
\text { e }\end{array}$ \\
\hline
\end{tabular}

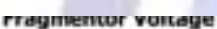

135
Comsion chergy
IOmazauon move

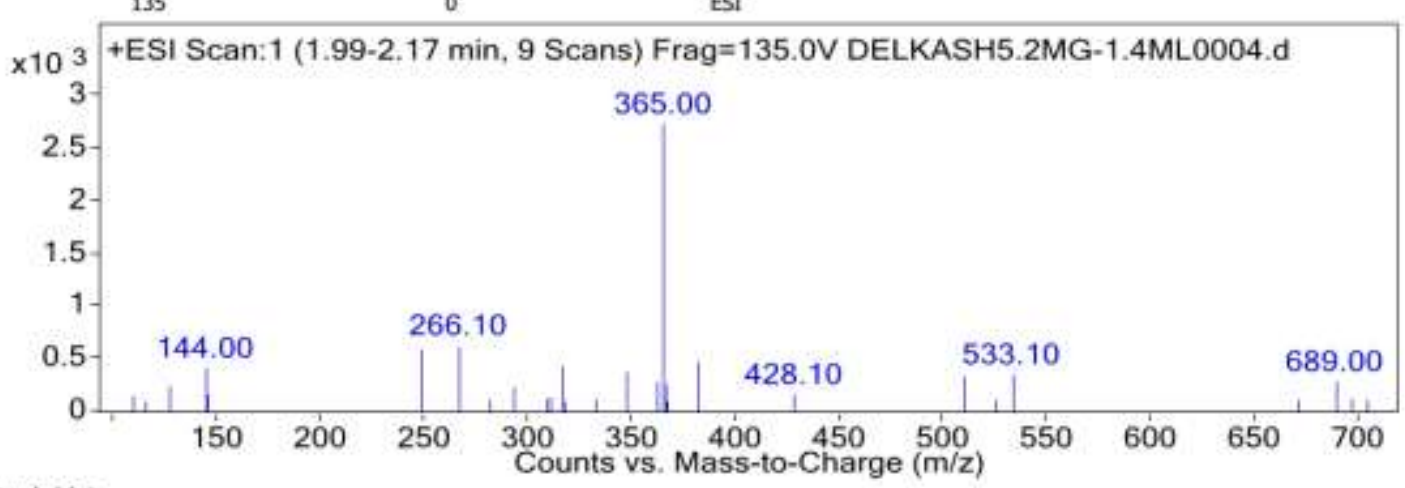

Peak List

\begin{tabular}{|l|l|}
\hline $\boldsymbol{m} / \boldsymbol{z}$ & Abund \\
\hline 144 & 411.9 \\
\hline 247.9 & 594.5 \\
\hline 266.1 & 625.7 \\
\hline 316 & 435.1 \\
\hline 347 & 387.1 \\
\hline 365 & 2733.6 \\
\hline 381.1 & 469.6 \\
\hline 509 & 330.1 \\
\hline 533.1 & 349.9 \\
\hline 689 & 282.7 \\
\hline
\end{tabular}



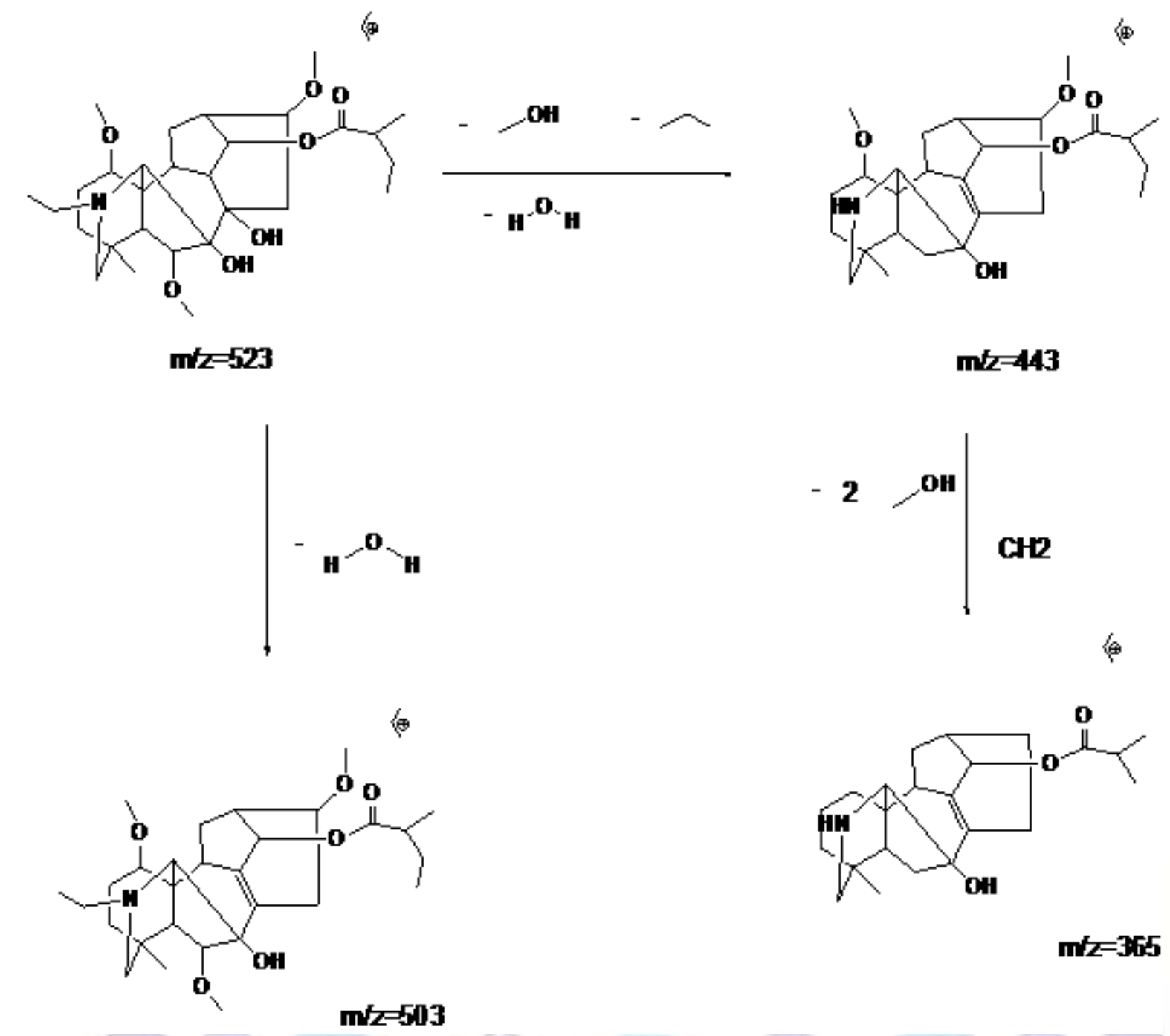

$m z=365$

Fragmentation pattern of 14-(2-methylbutyl nudicaudiline) 

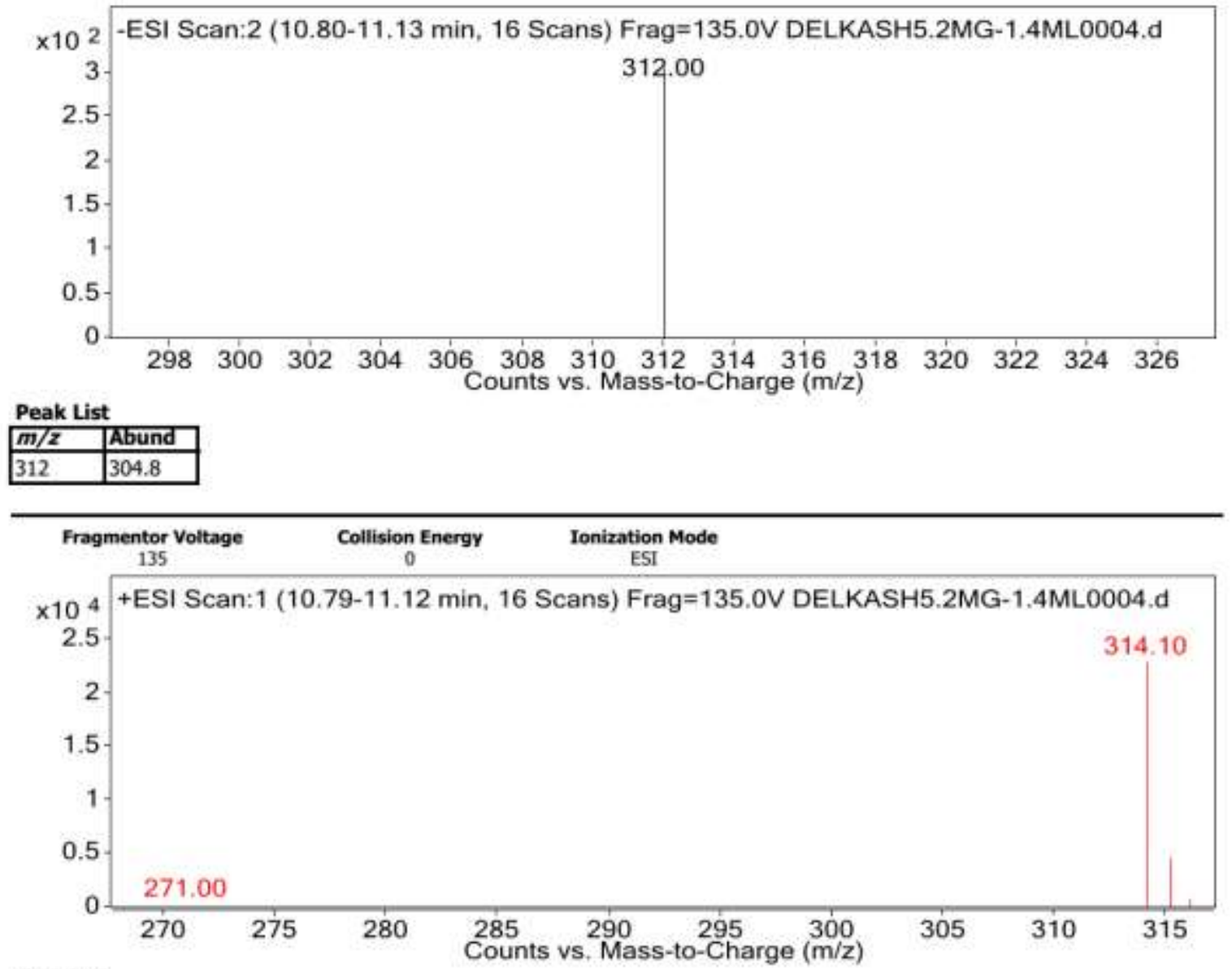

Peak List
\begin{tabular}{|l|l|}
\hline $\boldsymbol{m} / \boldsymbol{z}$ & Abund \\
\hline 268.9 & 136.6 \\
\hline 271 & 156.8 \\
\hline 313 & 142.3 \\
\hline 314.1 & 22814.2 \\
\hline 315.1 & 4681.3 \\
\hline 316 & 801.5 \\
\hline
\end{tabular}

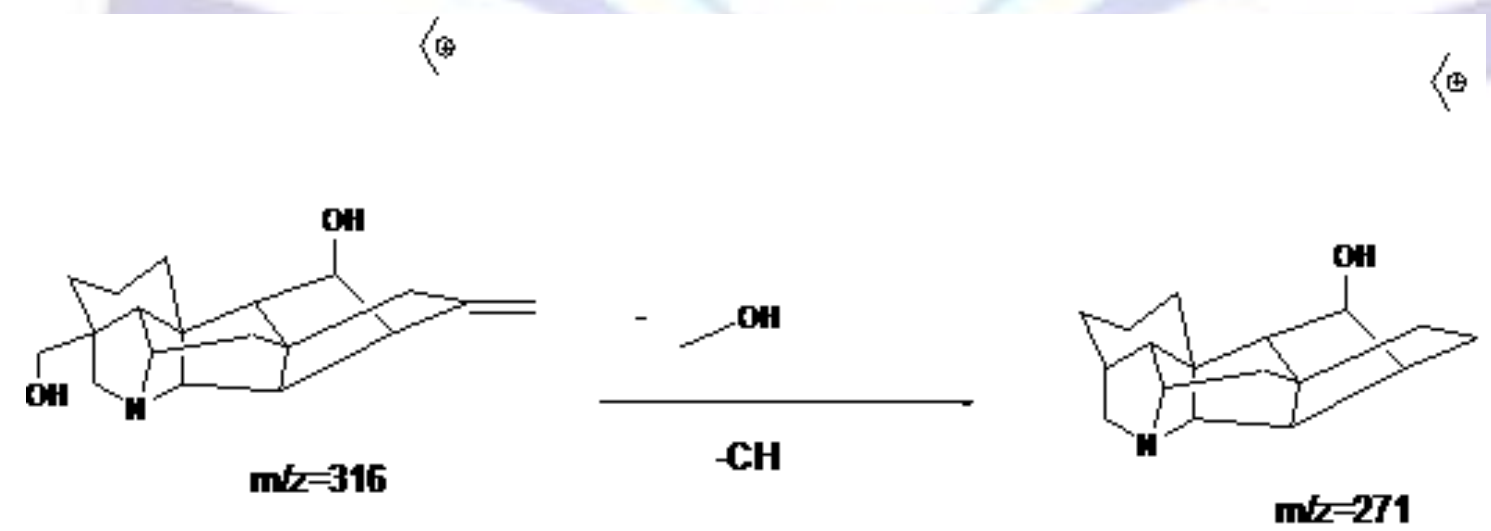

Fragmentation pattern of Davisinol 


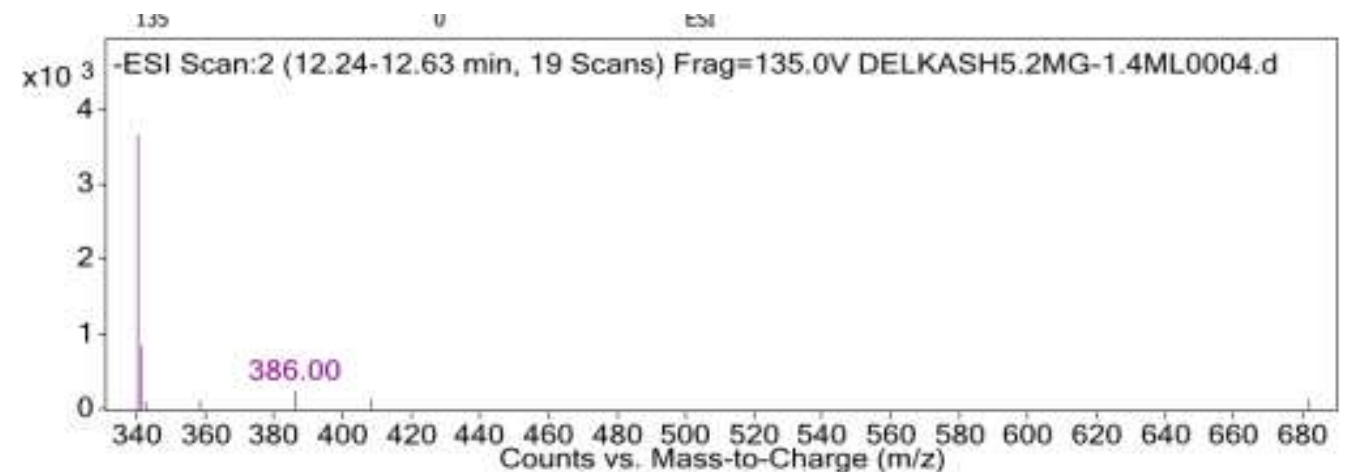

Peak List
\begin{tabular}{|l|l|}
\hline $\boldsymbol{m} / \boldsymbol{z}$ & Abund \\
\hline 340 & 3675.3 \\
\hline 341 & 851.3 \\
\hline 342 & 123 \\
\hline 357.9 & 145.9 \\
\hline 386 & 264.4 \\
\hline 407.9 & 163.7 \\
\hline 681.1 & 168.8 \\
\hline
\end{tabular}

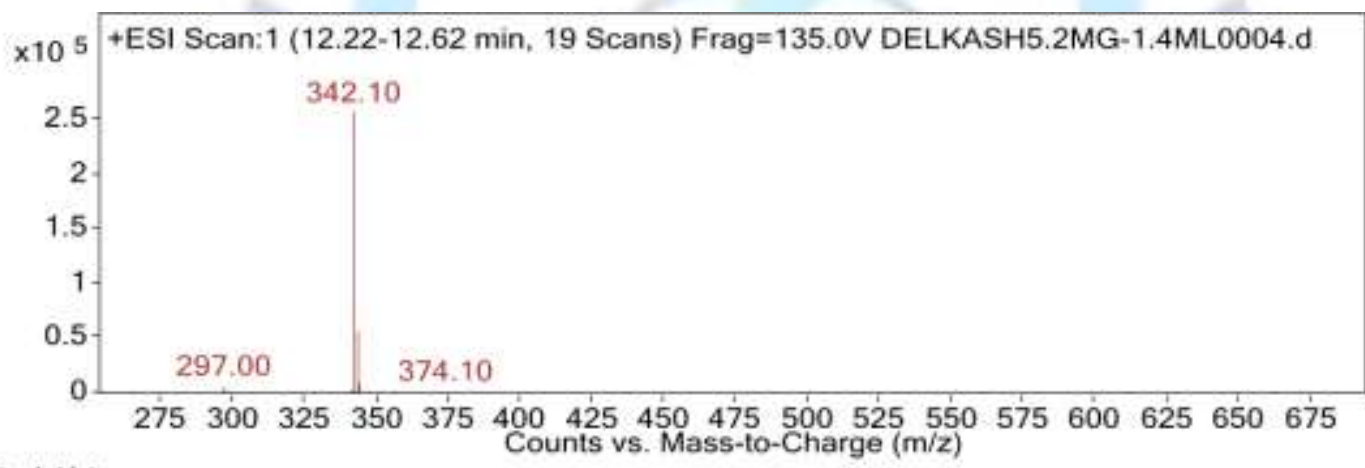

Peak List
\begin{tabular}{|l|l|}
\hline $\boldsymbol{m} / \boldsymbol{z}$ & Abund \\
\hline 265 & 1806.4 \\
\hline 297 & 6495 \\
\hline 328.1 & 2011.4 \\
\hline 341 & 2608.7 \\
\hline 342.1 & 256497.7 \\
\hline 343.1 & 56885.6 \\
\hline 344.1 & 9041.3 \\
\hline 356 & 1433.3 \\
\hline 374.1 & 1587.8 \\
\hline 683.1 & 1467.8 \\
\hline
\end{tabular}



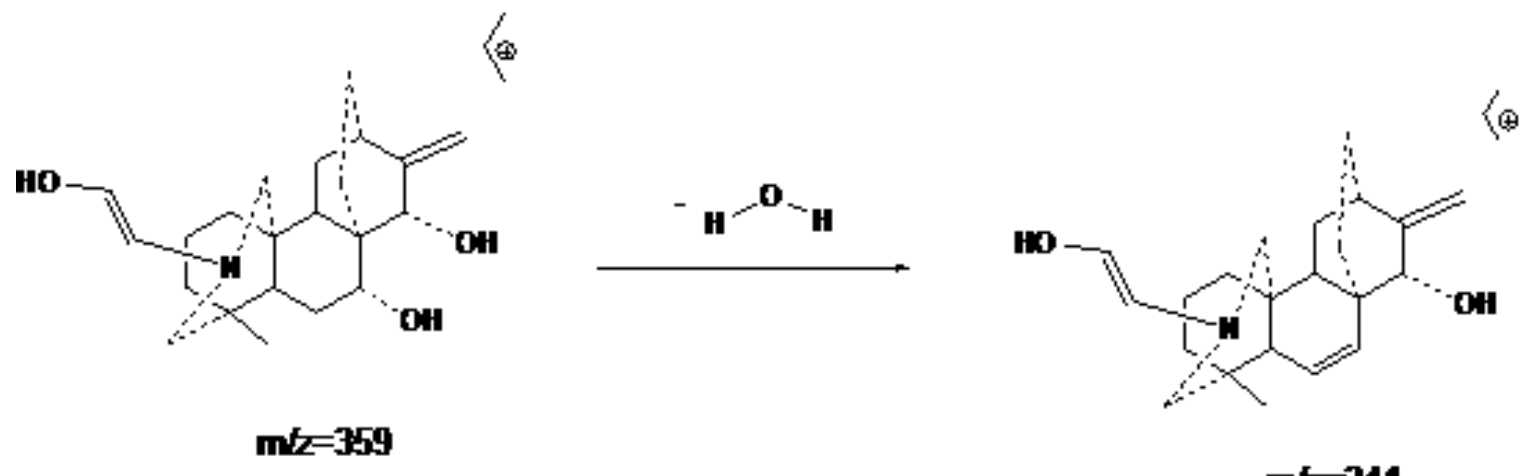

$$
m b=341
$$

Fragymentation patem of uniritie

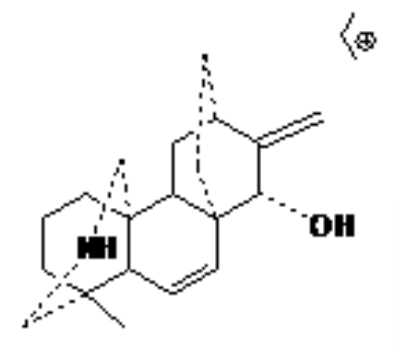

$m z=297$

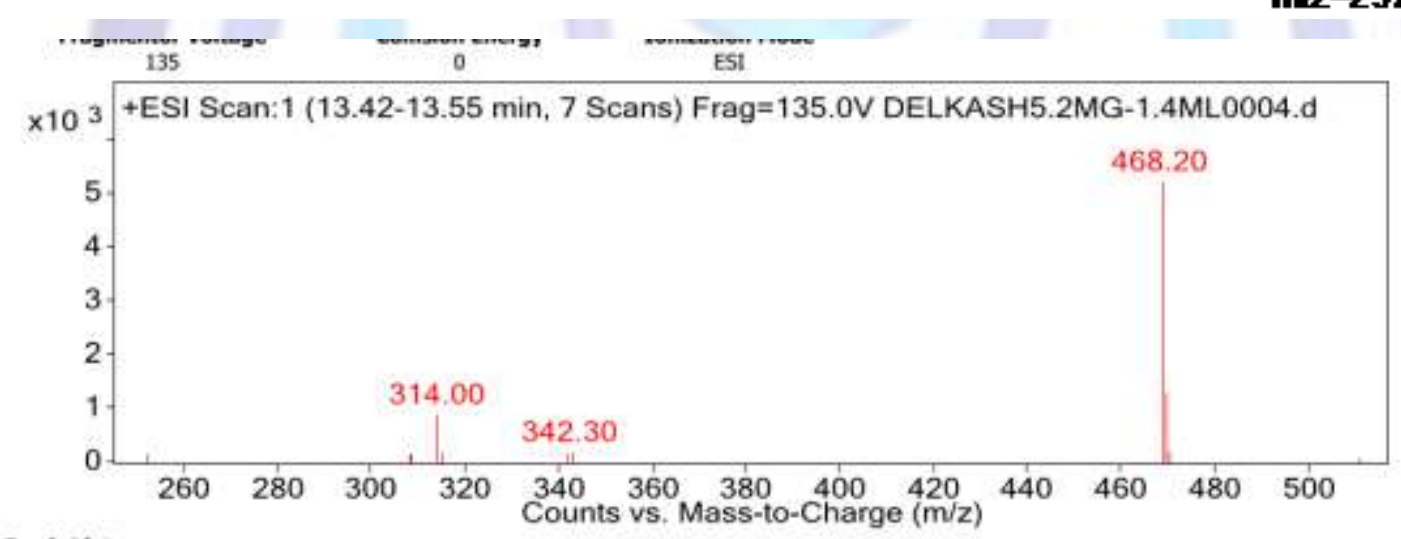

Peak List
\begin{tabular}{|l|l|}
\hline $\boldsymbol{m} / \boldsymbol{z}$ & Abund \\
\hline 252 & 146.4 \\
\hline 307.8 & 161.5 \\
\hline 308.3 & 146.9 \\
\hline 314 & 902 \\
\hline 315 & 199.6 \\
\hline 341.8 & 169.9 \\
\hline 342.3 & 202.1 \\
\hline 468.2 & 5234.4 \\
\hline 469.2 & 1329 \\
\hline 470 & 222.2 \\
\hline
\end{tabular}




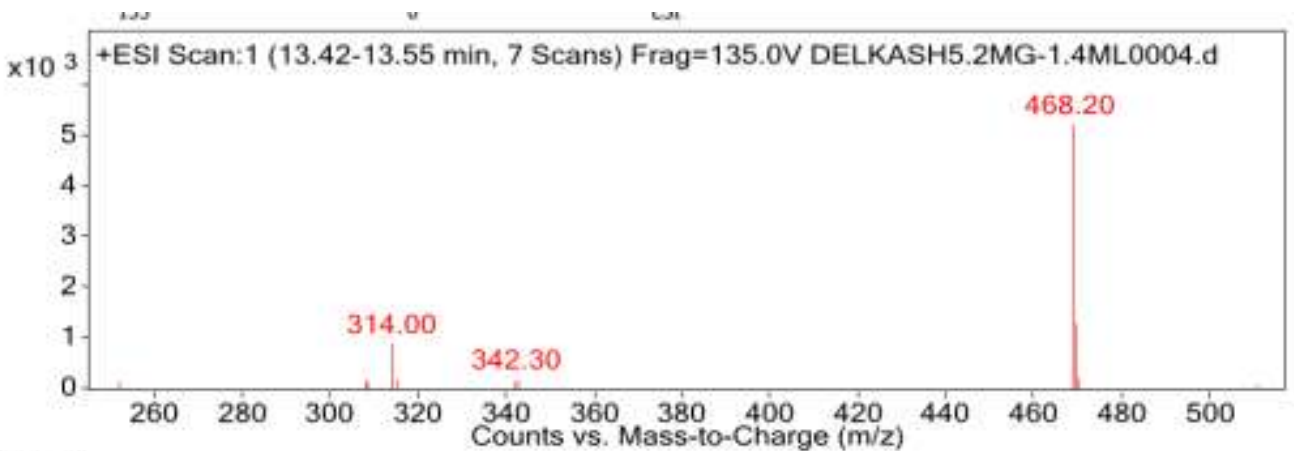

Peak List
\begin{tabular}{|l|l|}
\hline$m / z$ & Abund \\
\hline 252 & 146.4 \\
\hline 307.8 & 161.5 \\
\hline 308.3 & 146.9 \\
\hline 314 & 902 \\
\hline 315 & 199.6 \\
\hline 341.8 & 169.9 \\
\hline 342.3 & 202.1 \\
\hline 468.2 & 5234.4 \\
\hline 469.2 & 1329 \\
\hline 470 & 222.2 \\
\hline
\end{tabular}
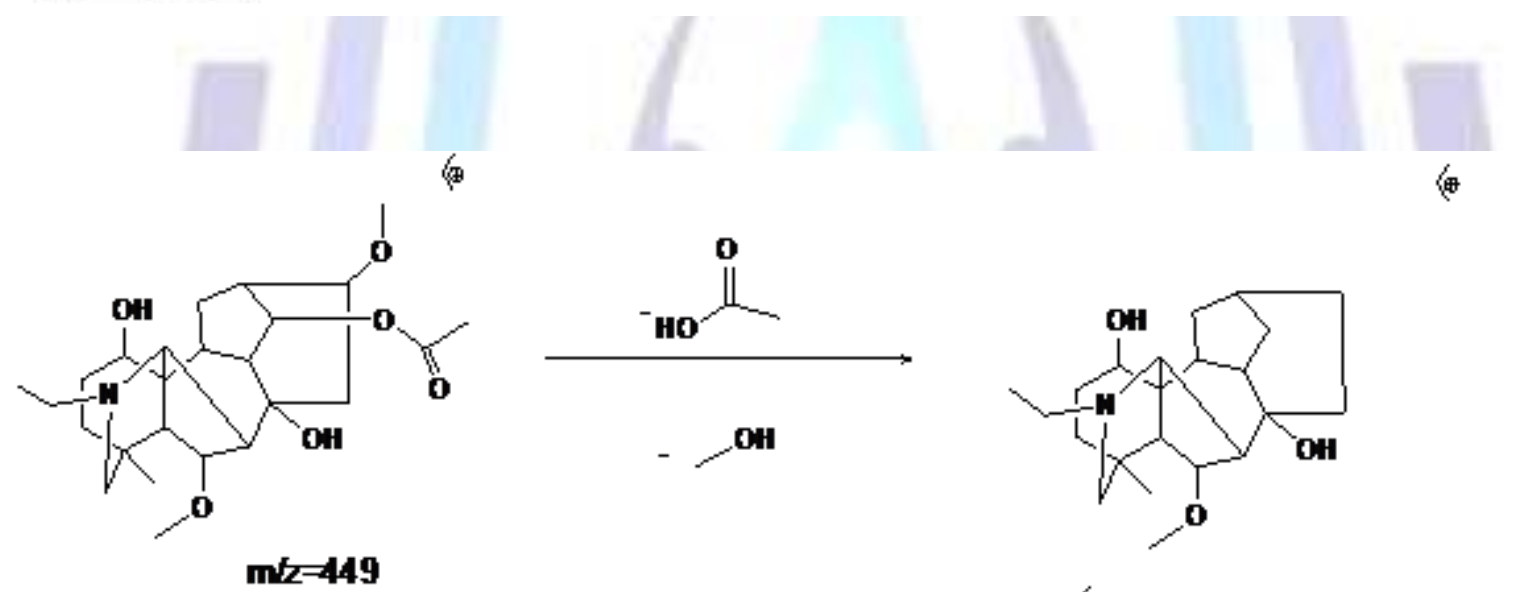

$m z-449$
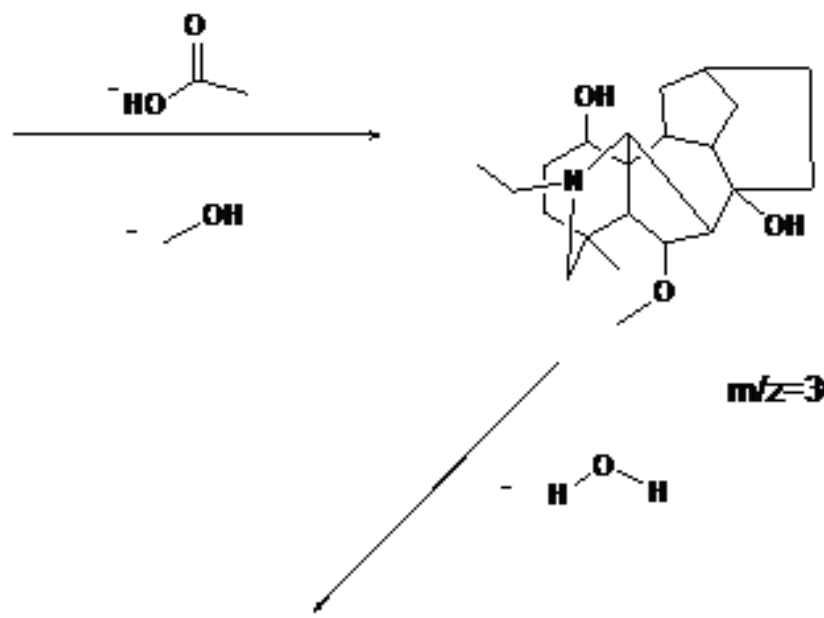

$m / 2=360$

便
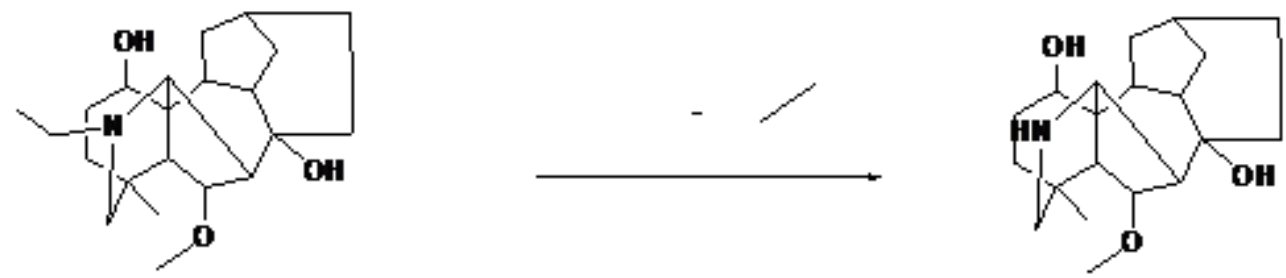

$m z=342$

$m / z=314$

Fragmentation pattern of condelphine 


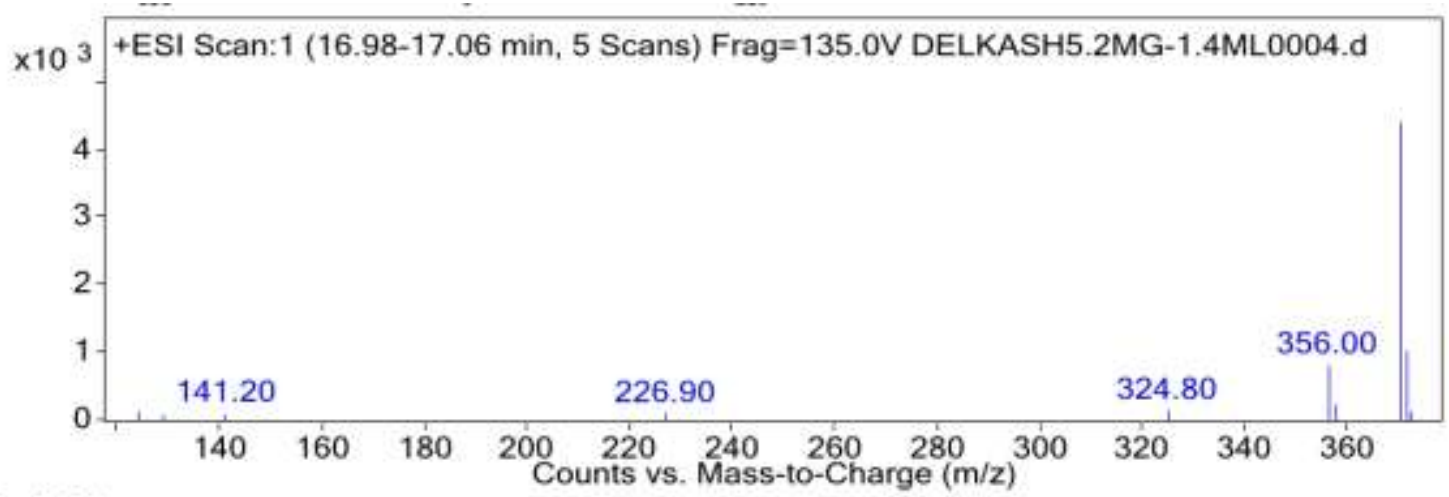

Peak List
\begin{tabular}{|l|l|}
\hline$m / z$ & Abund \\
\hline 124.2 & 123.2 \\
\hline 128.9 & 109.5 \\
\hline 141.2 & 119.1 \\
\hline 226.9 & 112.1 \\
\hline 324.8 & 157.6 \\
\hline 356 & 834.7 \\
\hline 357.1 & 246.2 \\
\hline 370.1 & 4413 \\
\hline 371.1 & 1027.8 \\
\hline 371.9 & 135.9 \\
\hline
\end{tabular}

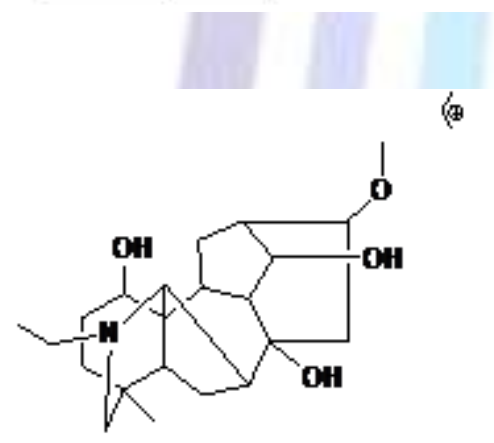

$m / z=374$

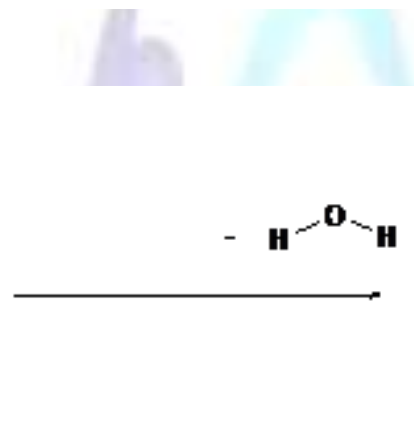

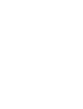
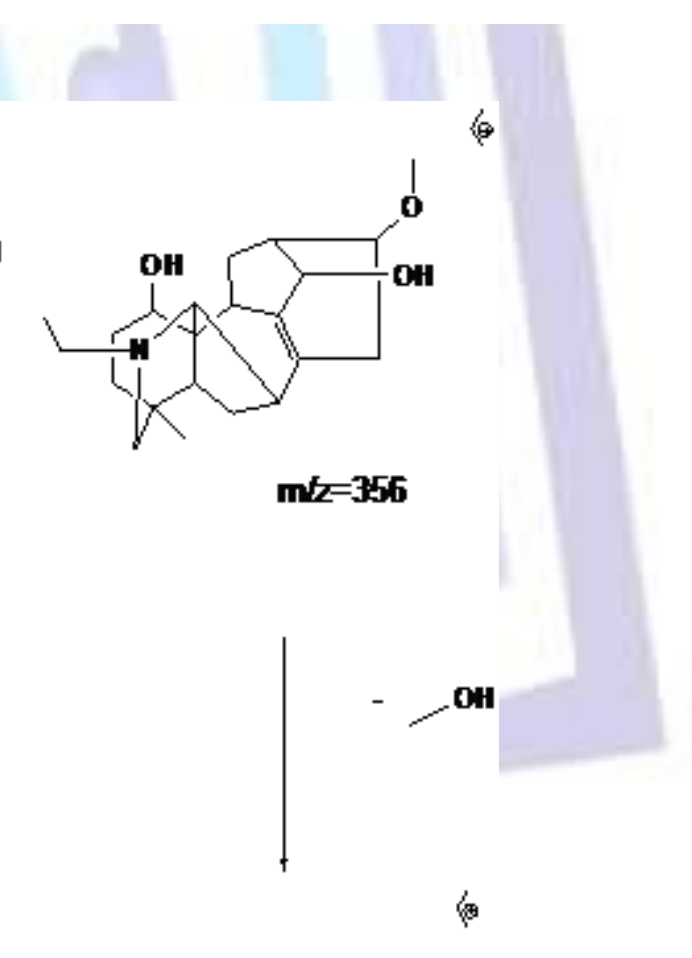

$<$

$$
m z=356
$$

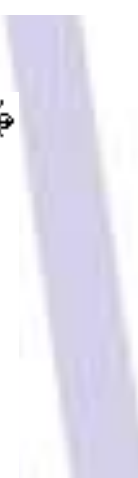

\section{Fragmentaion pateen of karaloline}




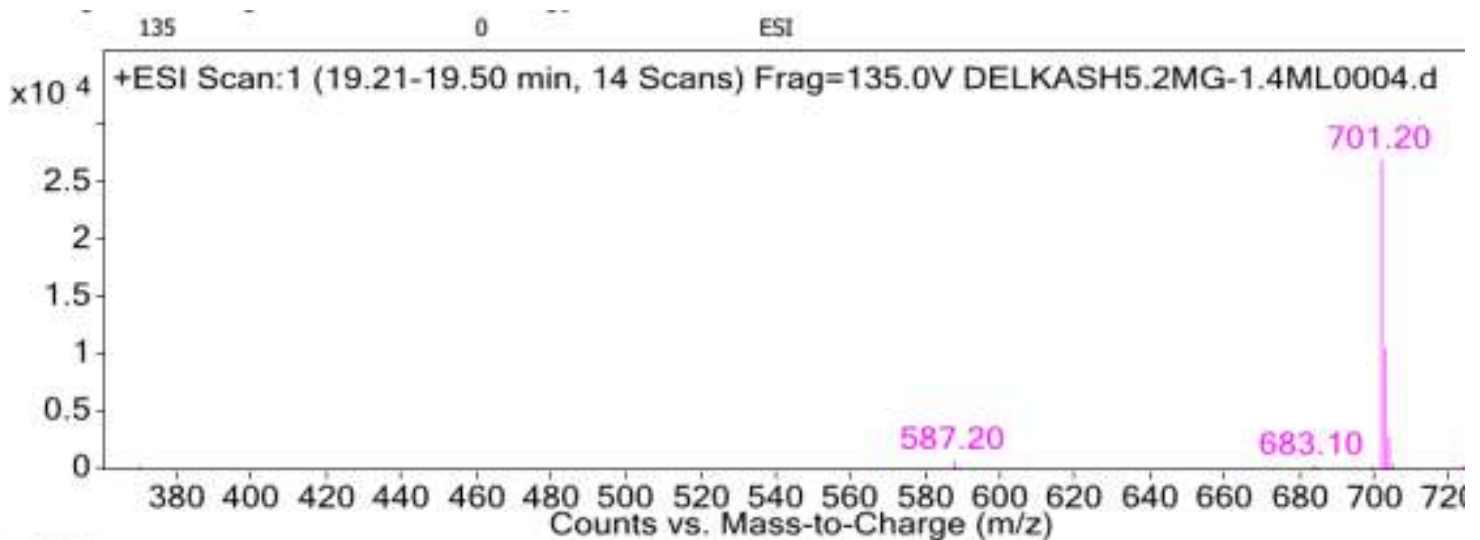

Peak List
\begin{tabular}{|l|l|}
\hline $\mathbf{m} / \boldsymbol{z}$ & Abund \\
\hline 370.1 & 375 \\
\hline 587.2 & 729.1 \\
\hline 683.1 & 256.9 \\
\hline 684.3 & 247.5 \\
\hline 699.1 & 370.7 \\
\hline 701.2 & 26960.8 \\
\hline 702.2 & 10627.9 \\
\hline 703.2 & 2835.8 \\
\hline 704.2 & 451.5 \\
\hline 723.1 & 302 \\
\hline
\end{tabular}




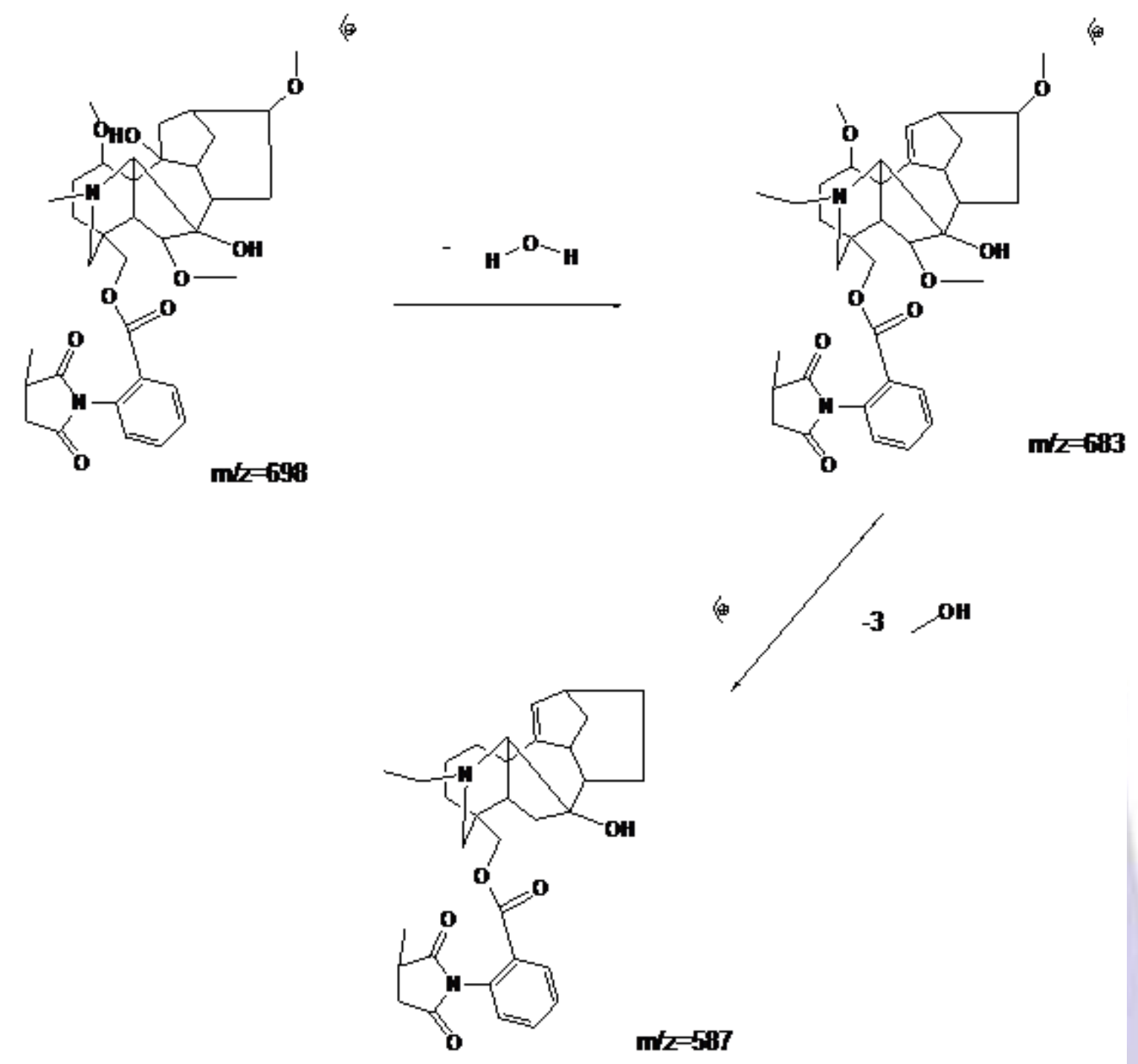

Fraymentation pattem of 10 hydroxymethylyoacontine 


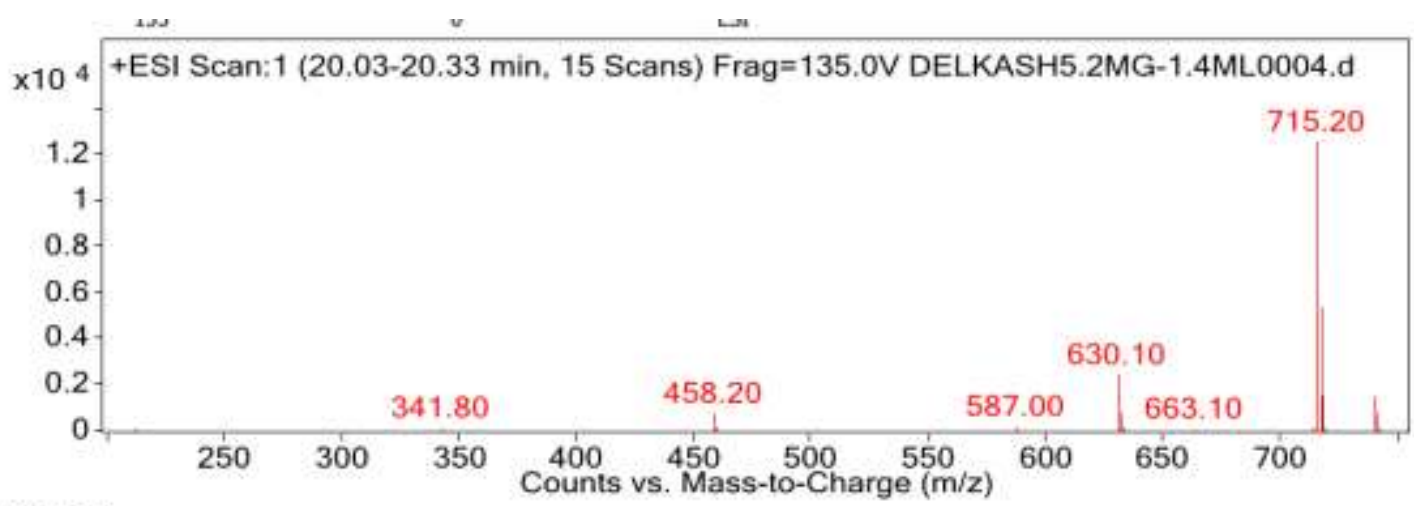

Peak List
\begin{tabular}{|l|l|}
\hline $\mathbf{m} / \boldsymbol{z}$ & Abund \\
\hline 458.2 & 770.8 \\
\hline 459.1 & 230.2 \\
\hline 630.1 & 2448.9 \\
\hline 631.2 & 848.8 \\
\hline 713.1 & 240.8 \\
\hline 715.2 & 12593.5 \\
\hline 716.3 & 5459.3 \\
\hline 717.2 & 1569.1 \\
\hline 739.2 & 1556.7 \\
\hline 740.2 & 828.4 \\
\hline
\end{tabular}

\section{CONCLUSION}

In this study a simple and efficient LC-ESI-MS ${ }^{n}$ method has been developed to identify alkaloids from roots of Delphinium cashmerianum. Moreover the LC-ESI-Mass spectrometry has been demonstrated to be an effective tool for analysis of the components and searching of novel compounds in plant extracts. It also provided the essential data for further phamacological and toxicological studies on Delphinium plants.

\section{REFERENCES}

1) Flora of North America: Delphinium

2) Sierra Nevada Wildflowers, Karen Wiese, 2013, p. 52

3) RHS A-Z encyclopedia of garden plants. United Kingdom: Dorling Kindersley. 2008. p. 1136. ISBN 1405332964

4) Jabbour, F., and S. S. Renner. 2011. Consolida and Aconitella are an annual clade of Delphinium (Ranunculaceae) that diversified in the Mediterranean basin and the Irano-Turanian region. Taxon 60(4): 1029-1040.

5) S. W. Pelletier, Z. Djarmati, S. Lasjic and Wilson, H. De Camp. University of Georgia, Athens, Georgia $30602,1975$.

6) Michael J. Warnock, Yong- Long Liu and Tom J. Mabry.Phytochemistry, 22, 1834-1835, 1983.

7) Ayhan Ulubelen, Jong C. Park, Ali H.Mericili and Filiz Mericili, Resit Ilarslan. J. Nat. Prod. 1996, 96. 360-366.

8) Martias Reina, Alberto Madinaveitia and Gabriel De La Fuente.Phytochemistry, 45, 1707-1711, 1997.

9) Ayhan Ulubelen, Muhammad arfan, Ufuk sonmez, Ali H Mercili, Filiz Mericili.Phytochem istry, 48, 385-388, 1998.

10) Nyamdari Batbayar, Shiter Enkhzaya, Jigidsuren Tusag, Dulamjav Batsuren, David S, Rycroft, Sussane Sproll, Franz Bracher. Phytochemistry, 62, 543-550, 2003.

11) Jesus G.Diaz, Armando J. Carmona, Pedro Perez de Paz, Werner Herz. Phytochemistry letters 1, 125-129, 2008.

12) Yang-Qing He, Zhan-Ying Ma, Qian Yang, Bao-Zhong Du, Zhan-Xin Jing, Bing-Hua Yao, Mark T. Hmann, Biochemical systematics and ecology 38, 554-556, 2010. 